\title{
Theoretical Study on Current-Voltage Characteristics of Oxygen Reduction Reaction near Platinum Surfaces
}

\author{
Sawanya Suwannawong, Kentaro Doi, and Satoyuki Kawano
}

\begin{abstract}
Recently, developments of alternative materials for energy sources are particularly required to overcome the environmental problems arising from the excessive use of fossil fuel. Proton-exchange membrane fuel cells (PEMFCs) are one of the most attractive research fields due to its capability of both stationary and portable applications at low working temperature. On the other hand, the low efficiency and high cost of PEMFCs prevent its commercial use. One of the serious problems is a catalytic activity of oxygen reduction reaction (ORR), which determines the slowest reaction rate. Herein, a previous theoretical study is applied to ORR near Pt surfaces in PEMFCs. The electronic structures of $\mathrm{Pt}$ clusters with an adsorbed $\mathrm{O}_{2}$ molecule are optimized by using the density functional theory (DFT) and $I-V$ characteristics are also analyzed. Our model can provide a different aspect of the dissociative adsorption process. The computational results are verified in comparison with the previous data.
\end{abstract}

Index Terms-Proton-exchange membrane fuel cells (PEMFCs), oxygen reduction reaction (ORR), I-V characteristics.

\section{INTRODUCTION}

Alternative energy technologies play an important role in recent energy crises. One of the most interests is proton exchange membrane fuel cells (PEMFCs), which generates electricity from chemical reactions with no pollutants except for water. However, the wide-scale application is limited because of the large overpotential and the high production cost in Pt-based catalysts. Therefore, this problem has been investigated in various research fields. An important issue for the wide use of PEMFCs is the improvement of the oxygen reduction reaction (ORR) which is the rate-determining step of overall electrochemical reactions. The ORR occurs at the cathode catalyst surfaces; thus, computations focusing on the interfaces, e.g., molecular dynamics (MD) simulations and $a b$-intio approaches, are also equally significant as well as the experiments [1]-[3]. Since the electron transfer is a vital component of the ORR, electronic structure calculations become a promising approach in the improvement of ORR activity. On the other hand, although this topic has been investigated by several computational methods, the variety of conclusions has not yet provided the common ground [1]-[3].

Previous studies have focused on the energy diagram, e.g., adsorption energy, Gibbs free energy, and potential energy surfaces, to identify the detail of ORR mechanism and

Manuscript received August 10, 2014; revised November 7, 2014.

Sawanya Suwannawong, Kentaro Doi, and Satoyuki Kawano are with the Department of Mechanical Science and Bioengineering, Graduate School of Engineering Science, Osaka University, 1-3 Machikaneyama, Toyonaka, Osaka, 560-8531, Japan (e-mail: kawano@me.es.osaka-u.ac.jp). compare the possibility of reaction pathways regarding the activation energies [1]. For example, Nørkskov et al. [4] introduced a simple method to calculate the stability of the reaction intermediates by generating the free-energy profile from the computed binding energy as a function of electrode potential $(U)$, by shifting the free energy of states containing by $-e U$, where $e$ is the electron charge. In that work, a slab model was produced to perform the electronic structure calculation. They suggested that the origin of overpotential was due to $\mathrm{O}$ and $\mathrm{OH}$ adsorption, while their association and dissociation might depend on the metal and applied potential. Although their work provided the useful results of the dissociative adsorption process considering the one-electron transfer, it was limited to describe the ionization potential $(I P)$ and electron affinity (EA) Due to time-consuming computations for large-sized electronic structures, effectively smaller scale models are thus required for more improvements. Panchenko et al. [5] carried out a density functional theory (DFT) calculation using a cluster model of $\mathrm{Pt}_{9}(111)$ and showed the capability of computing the adsorption energy, geometry, and the charge transfer in such a small-scale system.

In this study, the ORR occurring near the cathode surface is treated by our own method, according to a previous theoretical study [6], [7] in which a nonequilibrium current condition between a molecule and a couple of electrodes were investigated. By focusing on the interaction between molecules and catalytic materials, this model can be applied to analyze the current-voltage $(I-V)$ characteristics of PEMFCs associated with the overpotential. The first problem to handle the ORR with the ab initio approach is to model an effective structure of the surface. In terms of surface reaction systems, a catalyst material needs to be determined carefully, because previous experimental uses of explicitly preferred species, shapes, and arrangements of the materials to yield high performances have to be taken into consideration. Herein, we perform $a b$ initio computations to optimize electronic structures in a catalytic reaction associated with ORR, and apply our theoretical model to evaluate the $I-V$ characteristics. As a first step, planer platinum cluster models are computed to obtain the optimized electronic structures, varying structural parameters such as the number of Pt atoms. In the next step, the electrical characteristics will be introduced as an alternative method to understand the mechanism of ORR. The present model can evaluate electric current based on the atomistic interaction by using quantum mechanical approaches. Furthermore, it is found that atomistic interactions apparently affect the $I-V$ characteristics. This study will shed light on the non-equilibrium dynamics in chemical reaction systems. 


\section{THEORETICAL MODEL}

Dissociative adsorption of $\mathrm{O}_{2}$ molecules on a Pt surface can be compared to interactions between molecules and an electrode surface [8]. There are two important parameters: the degree of overlap of molecular orbitals (MOs) that describes the state of adsorption and the degree of charging that identifies the strength of coupling regarding the metal work function, electron affinity, and polarizability [9]. Such parameters are possibly represented by the induced dipole moment. To calculate a generated current due to the charge transfer between a metal cluster and an adsorbed molecule, overlaps of MOs and polarization should be effectively taken into account. We can apply a previous theoretical model to the present topic, which successfully explains the effects of charge transfer on tunneling current in a molecular junction [7]. To analyze the $I-V$ characteristics in molecules, the computation of electric current, $I$, is performed based on Heisenberg's uncertainty principle, such that,

$$
I \leq \frac{2 e \Delta N \Delta E}{\hbar}
$$

where $e$ is the electron charge, $\Delta N$ is the effective number of valence electron involved in the charge transfer, which can be computed by the relation of MO levels, and $\Delta E$ is the total energy change. $\Delta N$ can be computed by considering the effect of MO shifting due to the applied potential and the limitation owing to the energy gap of the highest occupied molecular orbital (HOMO) and the lowest unoccupied molecular orbital (LUMO). It can be numerically derived as follow:

$$
\Delta N=\frac{1}{4} \frac{\Delta I P+\Delta E A}{I P_{\mathrm{av}}-E A_{\mathrm{av}}}
$$

where $I P$ represents the ionization potential and $E A$ describes the electron affinity. $\triangle I P$ and $\triangle E A$ are calculated by the energy difference between a system with external potentials and that of equilibrium. $I P_{\mathrm{av}}$ and $E A_{\mathrm{av}}$ mean the average values of the system with and without applied electric field.

As mentioned above, coupling of the metal surface with a molecule can be estimated by the induced dipole moment; hence, $\Delta E$ in (1) is principally dominated by the polarization energy. Thereby, $\Delta E$ is approximately equivalent to the inner product of induced molecular dipole moment, $\Delta \vec{D}_{E}$, and the electric field, $\vec{E}$ :

$$
\Delta E=-\Delta \vec{D}_{E} \cdot \vec{E}
$$

In the ORR, since oxygen is reduced, electrons move from the $\mathrm{Pt}$ cluster to the $\mathrm{O}_{2}$ molecule. As a consequence, the voltage drop, $\Delta V$, is evaluated by the averaged potential difference between the $\mathrm{Pt}$ cluster and $\mathrm{O}_{2}$ molecule. It can be expressed as follows [10]:

$$
\begin{gathered}
V\left(\vec{R}_{A}\right)=\sum_{k \neq A} \frac{Z_{k} e}{4 \pi \varepsilon_{0}\left|\vec{R}_{A}-\vec{R}_{k}\right|}-\sum_{i j} \frac{e^{2} P_{i j}}{4 \pi \varepsilon_{0}} \int \frac{\phi_{i}(\vec{r}) \phi_{j}(\vec{r})}{\left|\vec{R}_{A}-\vec{r}\right|} d^{3} \vec{r} \\
\Delta V=\frac{1}{N} \sum_{i=1}^{N}\left(V_{E}\left(\vec{R}_{i}\right)-V_{0}\left(\vec{R}_{i}\right)\right)-\frac{1}{M} \sum_{i=1}^{M}\left(V_{E}\left(\vec{R}_{i}\right)-V_{0}\left(\vec{R}_{i}\right)\right)
\end{gathered}
$$

where $\varepsilon_{0}$ is the dielectric constant in vacuum, $N$ and $M$ represent the number of atoms in the $\mathrm{Pt}$ cluster and $\mathrm{O}_{2}$ molecules, respectively. $Z_{k}$ is the atomic number of the $k$ th atom, $\phi_{i}$ and $\phi_{j}$ are the orbital functions of the $i$ th and $j$ th level, respectively, and $P_{i j}$ is a corresponding element of density matrix. $\vec{R}_{A}, \vec{R}_{K}$, and $\vec{R}_{i}$ denote the position of nuclei. $V_{E}$ denotes the electrostatic potential at $\vec{R}_{i}$ in the electric field and $V_{0}$ denotes that of ground state.

\section{MethodOLOGY}

The electronic structure analysis is carried out using the Gaussian 09 program package [11]. The Becke three-parameter hybrid functional with the nonlocal correlation of Lee, Yang, and Parr (B3LYP) is employed for exchange-correlation interactions, using the double- $\zeta$ basis set with polarization functions of Weigend and Ahlrichs: Def2-SVP [12]. Varying the number of Pt atoms $n$, various sizes of $\mathrm{Pt}_{n}$ cluster are calculated to determine a preferable surface structure to simulate dissociative adsorption of $\mathrm{O}_{2}$ molecules near the surface. According to the optimized structure, a relationship between the response of electronic structures to electric fields and the $I-V$ characteristics is investigated, in which a $\mathrm{Pt}_{12}$ cluster interacts with an $\mathrm{O}_{2}$ molecule. Uniform electric fields with the intensity ranging from -0.001 to 0.001 a.u. are externally applied along the $z$-direction, which is perpendicular to the planer Pt cluster surface. According to the electronic structure calculations under the electric fields, the $I-V$ characteristics can be obtained by both the electric potential difference between a Pt cluster and an $\mathrm{O}_{2}$ molecule evaluated by (5) and the electric current by the maximum of (1).

\section{RESUlTS AND DISCUSSION}

\section{A. Optimization of Molecular Structures}

For computations within the theoretical framework [7], the initial configuration of $\mathrm{Pt}$ surface was presented by a planer monolayer. Separately optimized $\mathrm{Pt}_{n}$ clusters $(n=6,8,10$, and 12) were frozen and located on the $x y$-plane to additionally adsorb an $\mathrm{O}_{2}$ molecule on it. The $\mathrm{O}-\mathrm{O}$ bond lengths above $\mathrm{Pt}_{6}, \mathrm{Pt}_{8}, \mathrm{Pt}_{10}$, and $\mathrm{Pt}_{12}$ clusters resulted in 1.39, $1.33,1.28$, and $1.23 \AA$, respectively. Comparing to the natural bond length of $\mathrm{O}-\mathrm{O}$ in gas phase, $1.21 \AA$ [13], the bond length is elongated in all configurations. Although preferable structures of Pt planer surfaces were investigated up to $9 \mathrm{Pt}$ atoms [14], [15], calculations including an adsorbed $\mathrm{O}_{2}$ molecule shows that the adsorbate tends to interact with the Pt atoms at the edges. This may be caused by the crowded electron density due to the limitation of DFT method, so this inaccuracy should be improved by selecting the more suitable cluster size that is effectively comparable to the actual catalyst surface. The $\mathrm{Pt}_{12}$ cluster interacting with $\mathrm{O}_{2}$ near its center is shown in Fig. 1 (a). One of the $\mathrm{O}$ atoms located at $2.19 \AA$ near the top-site of a Pt atom. The other one was at the bridge site, being a little bit apart from the nearest one, where the $\mathrm{O}-\mathrm{O}$ length was $1.23 \AA$ and its bond axis tilted at $29.4^{\circ}$ to the surface. The adsorption energy $\left(E_{\mathrm{ad}}\right)$ was also analyzed as 
shown in Table I. The value resulted in $0.646 \mathrm{eV}$, which was similar to the previous experimental and computational results; hence, this approach was reasonable to represent the practical conditions. So as to indicate the charge transfer occurring in the ORR reaction, the induced charge density $(\Delta \rho)$ was calculated as;

$$
\Delta \rho=\rho\left(\mathrm{Pt}_{12} \mathrm{O}_{2}\right)-\rho\left(\mathrm{Pt}_{12}\right)-\rho\left(\mathrm{O}_{2}\right) .
$$

Fig. 2 presents the distribution of $\Delta \rho$ and describes that the $\mathrm{O}_{2}$ attracts electrons when it adsorbs on the $\mathrm{Pt}_{12}$ cluster. This is an essential process of the dissociative adsorption of $\mathrm{O}_{2}$ on metal surfaces. The higher density of electrons around the $\mathrm{O}_{2}$ molecule causes the repulsive interaction between each $\mathrm{O}$ atom and results in the bond elongation. Furthermore, the $I-V$ characteristics is another useful technique to explain the ORR mechanism in terms of electricity. According to the discussion above, hereafter the $\mathrm{Pt}_{12}$ cluster was used to carry out computations to treat charge transfer in the ORR.

\section{B. Effect of Position and Orientation of Adsorbed $\mathrm{O}_{2}$}

One of interesting issues in the reaction process on transition metals is adsorption sites on the catalyst surface. A previous study of ORR on $\mathrm{Pt}_{9}(111)$ concluded that the favorable position of oxygen adsorption were the hollow and bridge site [5]. Furthermore, the other previous calculation using a slab model also stated that $\mathrm{O}_{2}$ molecule tended to be adsorbed on these two sites [18]. Referring to the optimized structure and previous results, we investigate a relationship between the stable molecular structures and non-equilibrium current condition. To analyze the $I-V$ characteristics, a distance $d$ between an $\mathrm{O}_{2}$ molecule and the Pt surface were varied, considering both hollow and bridge sites as illustrated in Fig. 3. Moreover, the $\mathrm{O}_{2}$ was aligned in both perpendicular and parallel orientations to the Pt surface. Since the optimized results showed that the mass center of $\mathrm{O}_{2}$ adsorbed to the $\mathrm{Pt}$ surface at the bridge site horizontally, as shown in Fig. 1 (b) in which the angle of the $\mathrm{O}_{2}$ axis to the $x$-axis was $29.4^{\circ}$. Therefore, we focus on this configuration as a reference structure in the analysis of the HOMO.

(a)
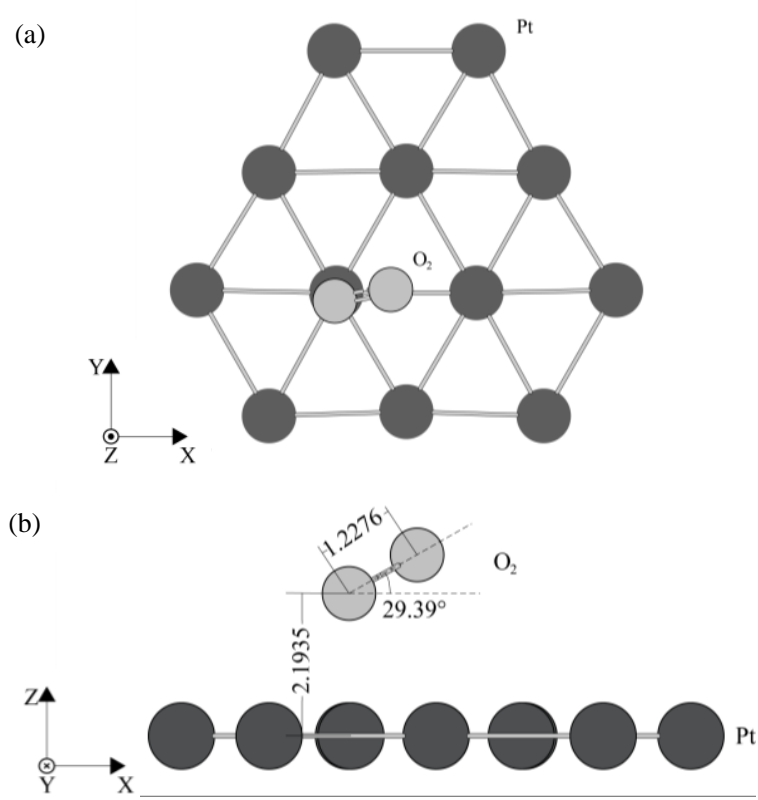

Fig. 1. Geometry of $\mathrm{Pt}_{12}$ cluster with adsorbed $\mathrm{O}_{2}$ in (a) the $x y$-plane and (b) the $x z$-plane.

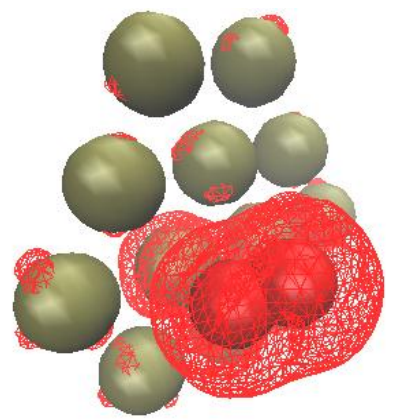

Fig. 2. Induced charge density due to the adsorption of $\mathrm{O}_{2}$ on the $\mathrm{Pt}$ surface.

TABLE I: THE DISSOCIATIVE ADSORPTION ENERGY

\begin{tabular}{ll}
\hline \hline Model and Functional & $E_{\mathrm{ad}}(\mathrm{eV})$ \\
\hline Experiment [16] & $\approx 0.5$ \\
$\mathrm{Pt}_{12}$ cluster model with B3LYP funcitonal & 0.646 \\
4-layer slab model with PW91 [5] & 0.63 \\
$\mathrm{Pt}_{1}$ to $\mathrm{Pt}_{6}$ cluster models with B3PW91 [17] & $0.53-0.83$ \\
\hline \hline
\end{tabular}

Figs. 4(a) and 4(b) show the $I-V$ characteristics of the bridge and hollow site with the parallel orientation of $\mathrm{O}_{2}$, respectively. Figs. 4(c) and 4(d) demonstrate the same procedure with the perpendicular orientation of $\mathrm{O}_{2}$. In all cases, for $d=5 \AA$, there seems to be higher conductance than the other case. This result indicates that although the interaction between the Pt cluster and $\mathrm{O}_{2}$ molecule may be weak, the dipole moment change is effective. Referring to a previous study [7], the large current at a long distance suggests a possibility of tunneling current due to the quantum effect. On the other hand, for $d=2$ through $4 \AA$, the conductance tends to be lower as $d$ decreases in all cases. This seems to be caused by the extremely strong coupling between the $\mathrm{Pt}$ and $\mathrm{O}_{2}$, in which the polarization become weak and the charge conduction will be disturbed.

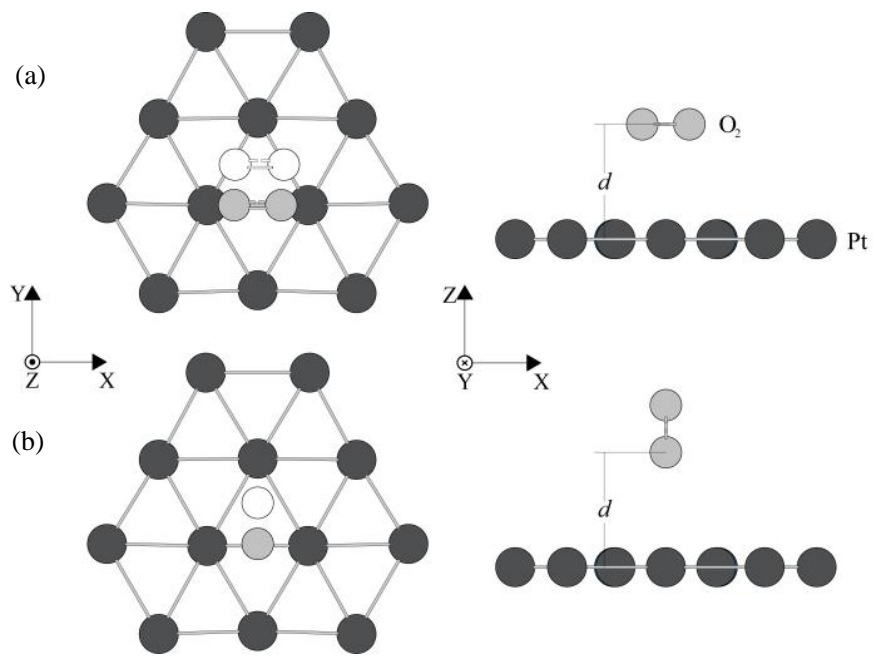

Fig. 3. Top-view and side-view of $\mathrm{Pt}_{12}$ cluster with (a) the parallel orientation of $\mathrm{O}_{2}$ at bridge (light gray) and hollow (white) site; (b) the perpendicular orientation at bridge (light gray) and hollow (white) site.

Fig. 5 shows the HOMO for the case of parallel orientation of $\mathrm{O}_{2}$ with $d=1 \AA$. Referring to the equilibrium condition of Fig. 5(a), it is found that the MO is affected by the electric field that is positive (Fig. 5(b)) and negative (Fig. 5(c)) direction along the $z$-axis. In this case, the HOMO broadly extends above both the $\mathrm{Pt}$ and $\mathrm{O}_{2}$ in which the electron tends to be bound in the complex and then, the response to the 
electric field is weak. On the other hand, as shown in Fig. 6 resulting from $d=3 \AA$, the HOMO clearly responds to the direction of electric field. In such a case, the effective conductance tends to be lower, since the electric potential difference becomes large due to the polarization against the electric current. Fig. 7 shows the result from $d=5 \AA$, in which the HOMO is localized only on Pt atoms and is not affected by the electric field. Due to the separation between the Pt cluster and $\mathrm{O}_{2}, \Delta V$ between the two is low and even small changes of $\Delta N$ and $\Delta E$ result in the high conductance. Fig. 8 presents the comparison of $I-V$ characteristics with respect to the orientations and the adsorption sites for the case of $d=3 \AA$. The results from the adsorption sites, i.e., hollow or bridge site, may not be clearly distinguished. On the other hand, the difference between the parallel and perpendicular orientation is clear, especially at positive voltages. The conductance of perpendicular orientation is higher than that of the parallel
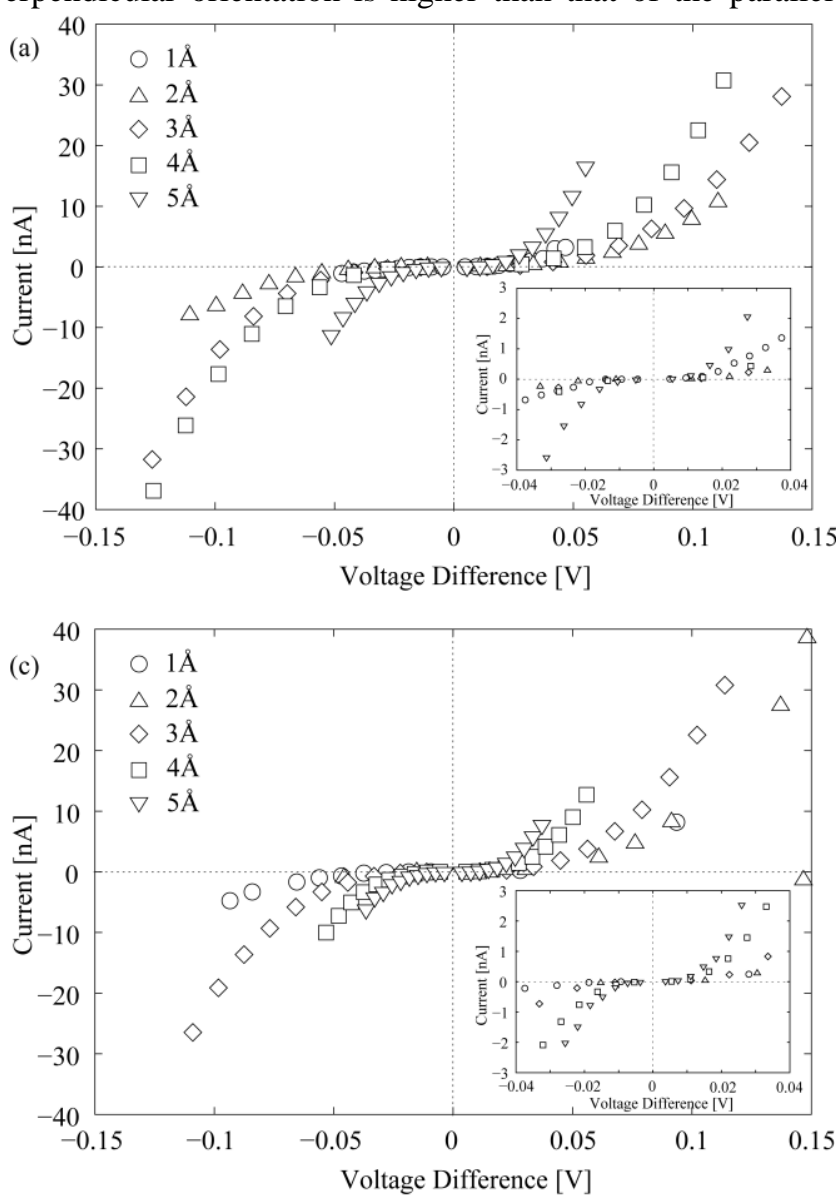

one. This demonstration indicates the degree of electric current in the complex system. It predicts the larger current when an adsorbed $\mathrm{O}_{2}$ is vertically aligned to the catalyst plane. That is, electrons on the Pt surface can easily transport to $\mathrm{O}_{2}$. From the energetical point of view, the parallel orientation at the bridge site was the most stable. This result indicates strong interactions between the two materials. On the other hand, in the $I-V$ characteristics, overlaps of MOs cause a strong polarization and generate a large potential difference in uniform electric fields, which result in the lower conductance. This is the correspondence relationship between the electronic structures and the $I-V$ characteristics. Furthermore, our results suggest that two stable states in equilibrium, such as a reactant and a product, have low conductance and that the transition between them in non-equilibrium conditions requires an overpontential to provide a constant electric current.
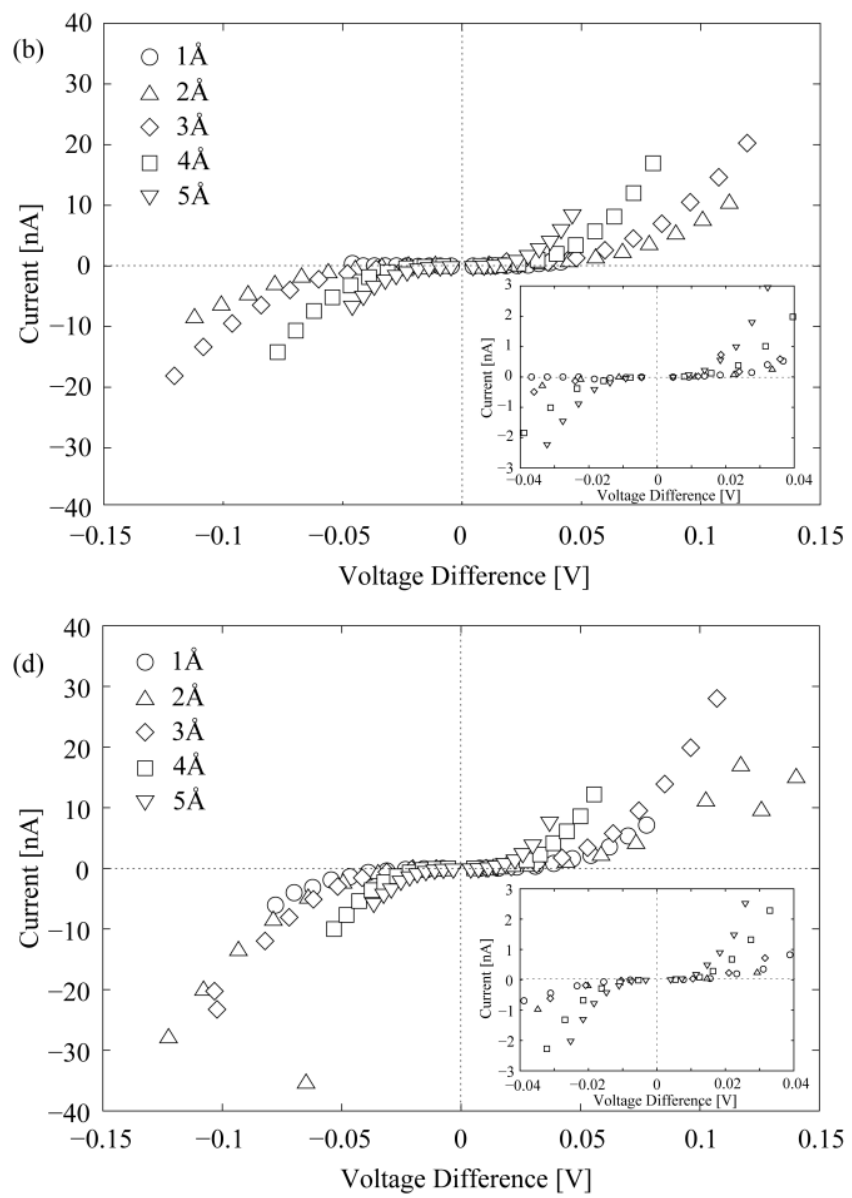

Fig. 4. I- $V$ characteristics for parallel orientation of $\mathrm{O}_{2}$ adsorbed at (a) bridge site and (b) hollow site as shown in Fig. 3; that for perpendicular orientation adsorbed at (c) bridge site and (d) hollow site. Magnified views ranging from -0.1 to $0.1 \mathrm{~V}$ are shown in insets.

(a)

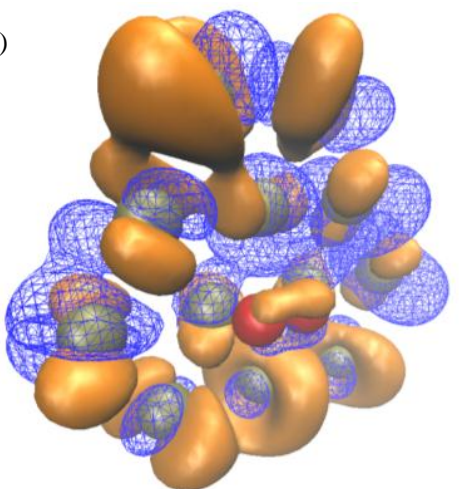

(b)

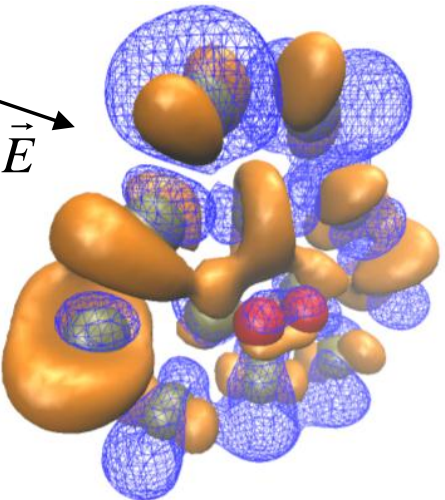

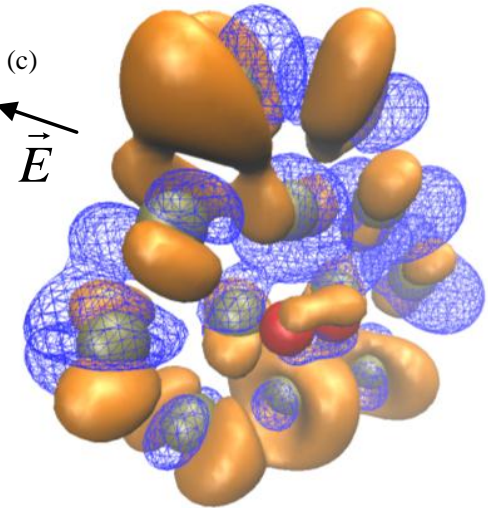

Fig. 5. Visualization of HOMO when $\mathrm{O}_{2}$ locate at $1 \AA$ from the Pt surface at the bridge site in parallel orienation, (a) without electric field, (b) with positive electric field, and (c) with negative electric field along the $z$-axis. MOs are presented at isovalue of \pm 0.01 (solid for positive and meshed for negative values). 

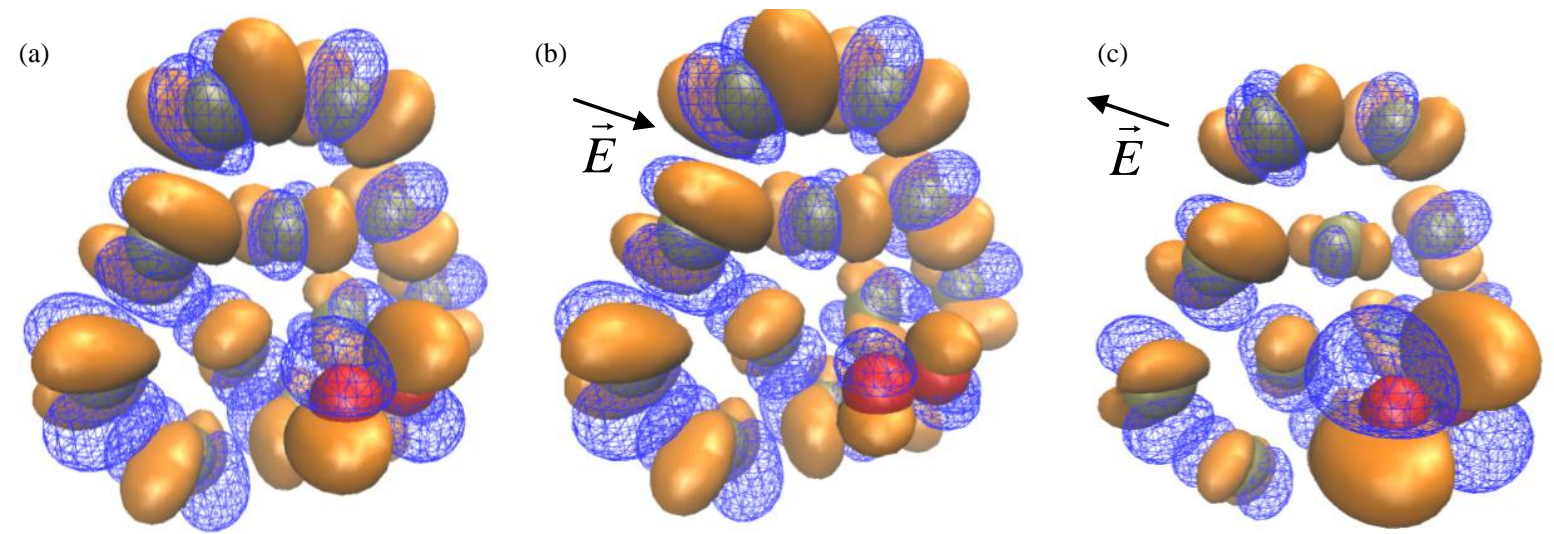

Fig. 6. Visualization of HOMO when $\mathrm{O}_{2}$ locates at $3 \AA$ from the Pt surface at the bridge site in parallel orientation, (a) without electric field,(b) with positive electric field, and (c) with negative electric field along the $z$-axis. MOs are presented in the same manner as Fig. 5.
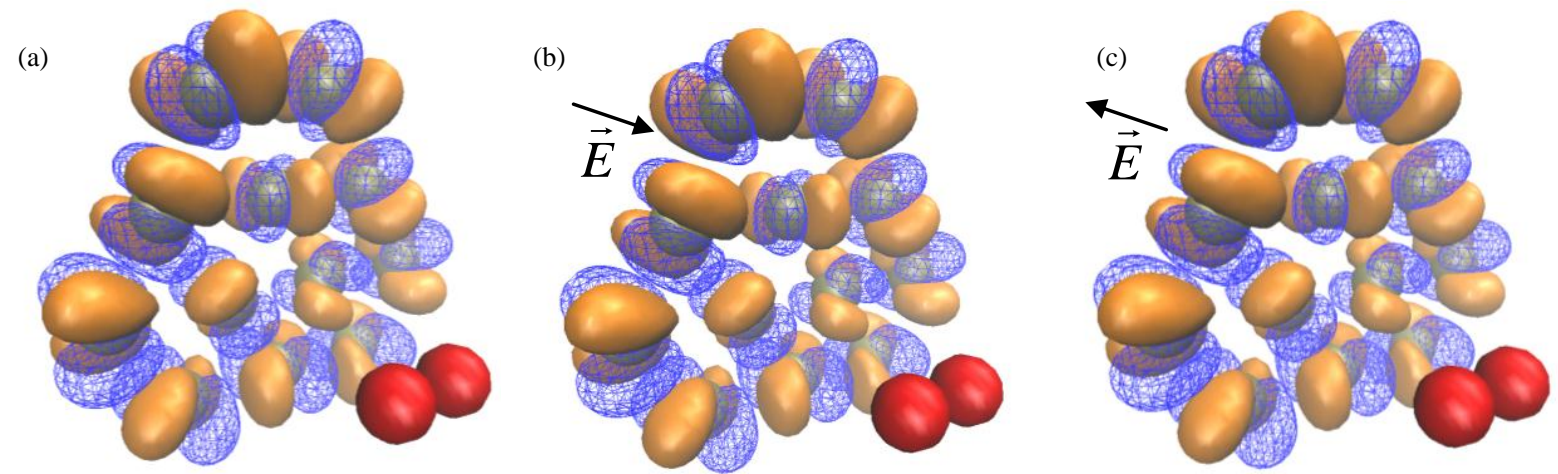

Fig. 7. Visualization of $\mathrm{HOMO}$ when $\mathrm{O}_{2}$ locates at $5 \AA$ from the Pt surface at the bridge site in parallel orientation, (a) without electric field, (b) with positive electric field, and (c) with negative electric field along the $z$-axis. MOs are presented in the same manner as Fig. 5.

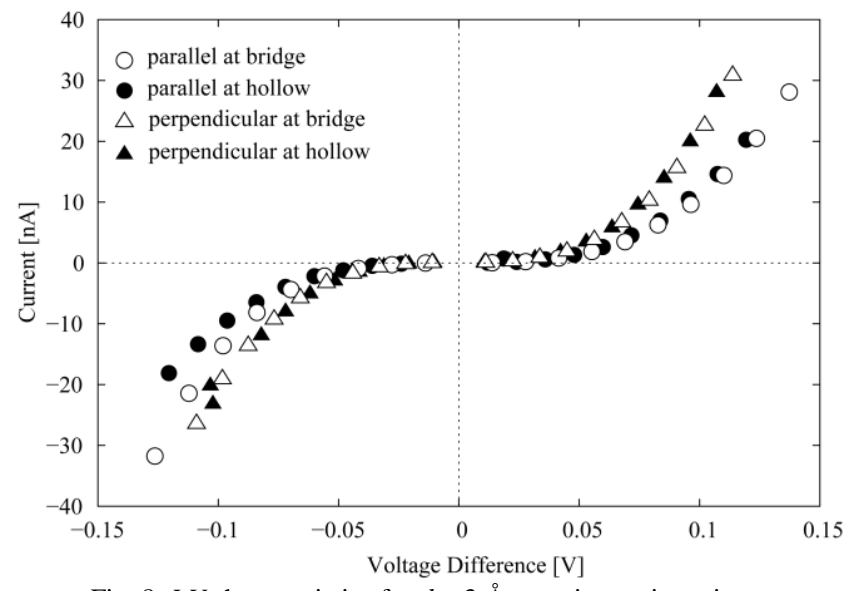

Fig. 8. I-V characteristics for $d=3 \AA$ at various orientations.

\section{CONCLUSION AND RECOMMENDATION}

The results of an optimized system of $\mathrm{Pt}_{n}$ clusters $(n=6$ to 12) and an $\mathrm{O}_{2}$ showed that a suitable structure for the theoretical model was determined as $\mathrm{Pt}_{12}$. The charge density was induced by the adsorption process and the $\mathrm{O}_{2}$ attracted electrons from the Pt cluster, which could indicate the trend of dissociative adsorption. $I-V$ characteristics were analyzed associated with the adsorption sites and orientations of an $\mathrm{O}_{2}$ molecule. Although there have been limitations in the model to quantitatively predict physical properties, differences in the geometrical configurations could be detected by the $I-V$ characteristics. The relationship between the MOs and $I-V$ characteristics could be discussed for ORR on the catalytic surface. In the future work, our method focusing on the frontier MOs will be applied, coupling with molecular dynamics simulations [19], [20], to electrochemical reactions at the electrode surfaces with regard to the ORR in PEMFCs.

\section{REFERENCES}

[1] C.-Y. Wang, "Fundamental models for fuel cell engineering," Chem. Rev., vol. 104, pp. 4727-4766, 2004.

[2] Z. Shi, J. Zhang, Z. S. Liu, H. Wang, and D. P. Wilkinson, "Current status of ab initio quantum chemistry study for oxygen electroreduction on fuel cell catalysts," Electrochim Acta, vol. 51, pp. 1905-1916, 2006.

[3] J. A. Keith and T. Jacob, "Theoretical studies of potential-dependent and competing mechanisms of the electrocatalytic oxygen reduction reaction on Pt(111)," Angew. Chem. Int. Edit, vol. 49, pp. 9521-9525, 2010.

[4] J. K. Nørskov et al., "Origin of the overpotential for oxygen reduction at a fuel-cell cathode," J. Phys. Chem. B, vol. 108, pp. 17886-17892, 2004.

[5] A. Panchenko, M. T. M. Koper, T. E. Shubina, S. J. Mitchell, and E. Roduner, "Ab initio calculations of Intermediates of oxygen reduction on low-index platinum surfaces," J. Electrochem. Soc., vol. 151, pp. A2016-A2027, 2004.

[6] K. Doi et al., "Ab initio calculation of electron effective masses in solid pentacene," J. Appl. Phys., vol. 98, p. 113709, 2005.

[7] P. Szarek, S. Suwannawong., K. Doi, and S. Kawano, "Theoretical study on physicochemical aspects of single molecular junction: application to the bases of ssDNA," J. Phys. Chem. C, vol. 117, pp. 10809-10817, 2013.

[8] W. Rainer, Nanoelectronics and Information Technology, 3rd ed., Wiley, 2012.

[9] K. Doi, I. Onishi, and S. Kawano, "Ab initio molecular dynamics of $\mathrm{H}_{2}$ dissociative adsorption on graphene surfaces," CMES, vol. 77, pp. 113-135, 2011.

[10] B. G. Johnson, P. M. W. Gill, J. A. Pople, and D. J. Fox, "Computing molecular electrostatic potentials with the PRISM algorithm," Chem. Phys. Lett., vol. 206, pp. 239-246, 1993.

[11] M. J. Frisch et al., Gaussian 09, Revision B.01, Gaussian, Inc., Wallingford CT., 2010.

[12] F. Weigend and R. Ahlrichs, "Balanced basis sets of split valence, triple zeta valence and quadruple zeta valence quality for $\mathrm{H}$ to $\mathrm{Rn}$ : 
Design and assessment of accuracy," Phys. Chem. Chem. Phys., vol. 7 , pp. 3297-3305, 2005.

[13] D. R. Lide, CRC Handbook of Chemistry and Physics, 86th ed., CRC Press, Boca Raton, FL, 2005.

[14] L. Xiao and L. Wang, "Structures of platinum clusters: planar or spherical?," J. Phys. Chem. A, vol. 108, pp. 8605-8614, 2004.

[15] V. Kumar and Y. Kawazoe, "Evolution of atomic and electronic structure of Pt clusters: Planar, layered, pyramidal, cage, cubic and octahedral growth," Phys. Rev. B, vol. 77, pp. 205418-1-205418-10, 2008.

[16] H. Steininger, S. Lehwald, and H. Ibach, "Adsorption of oxygen on Pt(111)," Surf. Sci., vol. 123, pp. 1-17, 1982.

[17] T. Li and P. B. Balbuena, "Computational studies of the interaction of oxygen with platinum clusters," J. Phys. Chem. B, vol. 105, pp. 9943-9952, 2001.

[18] A. Eichler and J. Hafner, "Molecular precursors in the dissociative adsorption of $\mathrm{O}_{2}$ on $\mathrm{Pt}(111)$," Phys. Rev. Lett., vol. 79, pp. 4481-4484, 1997.

[19] H. Nakano, H. Ohta, A. Yokoe, K. Doi, and A. Tachibana, "First-principle molecular-dynamics study of hydrogen adsorption on an aluminum-doped carbon nanotube," J. Power Sources, vol. 163, pp. 125-134, 2006.

[20] K. Doi, H. Nakano, H. Ohta, and A. Tachibana, "First-principle molecular-dynamics study of hydrogen and aluminum nanowires in carbon nanotubes," Materials Science Forum, vol. 539-543, pp. 1409-1414, 2007.

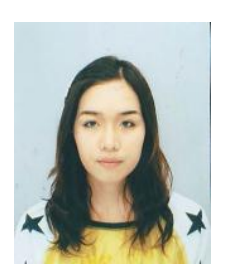

Sawanya Suwannawong is presently doing her $\mathrm{PhD}$ in Prof. Kawano Satoyuki`s Research Laboratory, at the Graduate School of Engineering Science, Osaka University. She completed her master course in 2013 on the Ab initio approach to study the electron transport in single-molecule junctions. Her current research interests are in improving the $\mathrm{Ab}$ initio approach and coupling with molecular dynamic to study the phenomena of charge transfer and electrochemical reaction on catalyst layer of PEMFCs. 